\title{
Metodologías Sugeridas de Evaluación y Selección de Software de Arquitectura Empresarial para la Digitalización del Conocimiento
}

\section{(Suggested Methodologies for Evaluation and Selection of Enterprise Architecture Software for Knowledge Digitization)}

\author{
Oswaldo Moscoso-Zea', Sergio Lujan-Mora²
}

\begin{abstract}
Resumen:
La gestión del conocimiento (GC) es una práctica de administración que permite la creación, uso, distribución y transferencia del conocimiento en organizaciones. Existen marcos de referencia de GC que incluyen componentes de inteligencia de negocios o de arquitectura empresarial (AE) para la implementación de proyectos de GC en organizaciones. Las herramientas de $\mathrm{AE}$ son usadas para digitalizar, relacionar y visualizar varias dimensiones de conocimiento como son: estructura organizacional, procesos de negocio, aplicaciones e infraestructura tecnológica. Este artículo tiene como objetivo evaluar el rol de la $A E$ como componente clave de la GC y sugerir metodologías que pueden ser usadas para evaluar herramientas de software de AE. Para esto se realizó una revisión bibliográfica de las metodologías de evaluación de software existente en el mercado y se eligieron aquellas que pueden ser adaptadas para el campo de la AE. Está investigación fue cualitativa y exploratoria a través de un caso de estudio desarrollado en un proveedor de servicios logísticos con presencia internacional. El caso de estudio muestra el proceso realizado para elegir la metodología de evaluación. Además, se describen los pasos para la digitalización del conocimiento organizacional.
\end{abstract}

Palabras clave: Gestión del Conocimiento; Arquitectura Empresarial, Metodología de Evaluación de Herramientas de Arquitectura Empresarial.

\begin{abstract}
:
Knowledge Management (KM) is a practice that allows the creation, use, distribution and transfer of knowledge in organizations. Different KM frameworks exist that include business intelligence or enterprise architecture (EA) components for the implementation of $\mathrm{KM}$ in organizations. EA tools are used to digitize, relate and visualize the following dimensions of knowledge: organizational structure, business processes, applications and technology. The objective of this paper is to assess the role of EA as a key component in KM and to suggest software evaluation methodologies that can be adapted for the field of EA. For this, an investigation was realized to identify the existing software evaluation methodologies in the market and to filter those that can be adapted for the field of EA. The methodology used for the research was qualitative and exploratory using a case study performed in an international logistic service provider. The case study describes the process done for the selection of the evaluation methodology. Furthermore, it describes the steps for knowledge digitization.
\end{abstract}

Keywords: Knowledge Management; Enterprise Architecture; Evaluation of Enterprise Architecture Tools.

\footnotetext{
1 Universidad Tecnológica Equinoccial, Quito - Ecuador (omoscoso@ute.edu.ec)

2 Universidad Alicante, Alicante - España (sergio.lujan@ua.es)
} 


\section{Introducción}

La innovación en la ciencia y en la tecnología ha dado lugar a organizaciones sólidamente basadas en la información. Estas organizaciones requieren transformar esta información en conocimiento para asegurar su competitividad y para mejorar la toma de decisiones. La gestión del conocimiento (GC) es una disciplina en evolución que apoya en la creación, uso, distribución y transferencia del conocimiento en las organizaciones (Campbell, 2006).

Las principales dimensiones que se deben analizar en un proyecto de GC son las personas, los procesos y la tecnología (Edwards, 2011). En un estudio previo realizado como parte de un proyecto de investigación, se propuso un marco de referencia de GC (Moscoso-Zea, Luján-Mora, Schweimanns, \& Esquetini, 2016). Este marco de referencia fue creado con el propósito de guiar y conducir la implementación de proyectos de GC en organizaciones, para asegurar la captura del conocimiento implícito y explícito de las tres dimensiones previamente mencionadas.

Lo innovador de este marco es que incorpora dos prácticas de gestión como son la inteligencia de negocios y la arquitectura empresarial (AE). En este artículo nos centraremos en revisar el rol de la $A E$ como un componente clave para la $G C$ y detallaremos cuáles son las herramientas tecnológicas de $\mathrm{AE}$ que existen en el mercado. Además se propone un enfoque de evaluación y selección de herramientas de $\mathrm{AE}$. En los últimos años la $\mathrm{AE}$ se ha convertido en una de las temáticas más importantes a considerar en los estudios de sistemas de información y ha evolucionado hasta convertirse en una tarea esencial de gestión de negocios, por lo cual es vital un análisis más profundo (Bricknal, Darrell, Nilsson, \& Pessi, 2011).

El artículo está estructurado de la siguiente forma. En la sección 2 se define la GC, la AE y las herramientas de $A E$. En la sección 3 se presentan los resultados de la investigación con un caso de estudio realizado en una organización internacional privada. El caso de estudio resalta como la AE puede apoyar a la GC y como se debería llevar un proceso de selección y evaluación de software o de herramientas de AE. Finalmente, se presentan las conclusiones de la investigación en la sección 4.

\section{Marco teórico}

En esta sección se presenta la investigación bibliográfica realizada para este trabajo.

\subsection{Gestión del conocimiento}

El conocimiento es uno de los recursos claves que puede fortalecer el posicionamiento de una organización (Curado, 2006). Un recurso debe ser valioso, raro e imperfectamente imitable con el fin de que la organización pueda mantener una ventaja competitiva (Wernerfelt, 1984). E conocimiento organizacional cumple con estas características; por lo tanto, debería ser recolectado y gestionado eficientemente. El conocimiento puede ser definido como experiencias, 
hechos, procesos y creencias que incrementan las capacidades individuales $u$ organizacionales (Karemente, Aduwo, Mugejjera, \& Lubega, 2009).

La GC es "el proceso de identificar, recolectar y aprovechar el conocimiento colectivo de una organización para potenciar a que la organización compita" (Alavi \& Leidner, 2001). Adicionalmente, la GC "se ocupa de la explotación y el desarrollo de los activos de conocimiento de una organización con el fin de cumplir con los objetivos de la organización" (Rowley, 2000). Las razones críticas para realizar GC son: rotación de personal, sobrecarga de información, incremento en la necesidad de personal experto, mejoramiento en la toma de decisiones y digitalización del conocimiento organizacional.

De las definiciones se derivan dos tareas esenciales que son necesarias para implementar la GC. Primero, se necesita establecer la infraestructura tecnológica que facilite capturar y compartir el conocimiento; segundo, se requiere establecer mecanismos y procedimientos para digitalizar el conocimiento tácito de las personas y los procesos. Estas dos tareas abarcan las dimensiones del conocimiento que son: personas, procesos y tecnología.

\subsection{Arquitectura empresarial}

En la actualidad no existe unanimidad en la definición de AE. Una de las razonas para que no exista acuerdo en una definición estándar podría ser porque la práctica de $\mathrm{AE}$ se encuentra todavía en una etapa de evolución (Ivanov, 2009). De acuerdo a Lankhorst (2009) "AE es un conjunto coherente de principios, métodos y modelos que son usados en el diseño, realización y mantenimiento de las arquitecturas de negocio, estructura organizacional, arquitectura de información y arquitectura tecnológica con respecto a la estrategia corporativa". Otra definición de AE es dada por Gartner (2013) "AE es el proceso de traducir la visión del negocio y su estrategia en un cambio empresarial efectivo por medio de crear, comunicar y mejorar los requerimientos clave, los principios y modelos que describen el estado futuro de la empresa y permiten su evolución". Esta es la definición que usaremos en este documento.

Como vemos en las dos definiciones, la $A E$ va más allá de la alineación de las tecnologías de información con el negocio. AE se refiere a la necesidad de gestionar la creciente complejidad organizacional y el cambio continuo, proveyendo una visión holística de la organización. La AE se la considera como una herramienta de gestión crucial para la alta dirección de las empresas. La $A E$ permite que los ejecutivos asuman su responsabilidad en el manejo, control y guía en las transformaciones basados en la visibilidad y evidencia que esta otorga (Proper \& Lankhorst, 2014). Además, la $A E$ permite mejorar el desempeño de las organizaciones ya que resuelve el problema de otorgar valor a través de sus sistemas de información (de Vries \& van Rensburg, 2008). La Figura 1 muestra una pirámide con los niveles organizacionales (dominios empresariales) como son: gente, aplicaciones, negocio y tecnología. Las flechas circulares 
secuenciales describen el proceso para implementar AE: obtener la aceptación y el apoyo ejecutivo, establecer una estructura de gestión y control, definir el enfoque de arquitectura, desarrollar la línea base y futura de AE, desarrollar el plan de secuencia, uso y mantenimiento de $\mathrm{AE}$.

Uno de los elementos clave para la implementación de AE es el uso de un marco de arquitectura. El organismo The Open Group (2011) describe muy bien a un marco de arquitectura como: "la estructura base o un conjunto de estructuras que pueden ser usadas para desarrollar distintos tipos de arquitecturas. Debe describir un método para diseñar el estado futuro de una organización en términos de un conjunto de bloques de construcción, y para indicar como estos bloques de construcción interactúan juntos. Debería contener un conjunto de herramientas que otorgan un vocabulario en común. También debería contener una lista de estándares recomendados y productos compatibles que se pueden usar para implementar los bloques de construcción". Un framework sirve para guiar el esfuerzo (operacional) o para estructurar la información organizacional (conceptual) (D. Matthes, 2011). Como se puede ver, los marcos de AE proveen un conjunto de guías y mejores prácticas para la implementación y mantenimiento de AE.

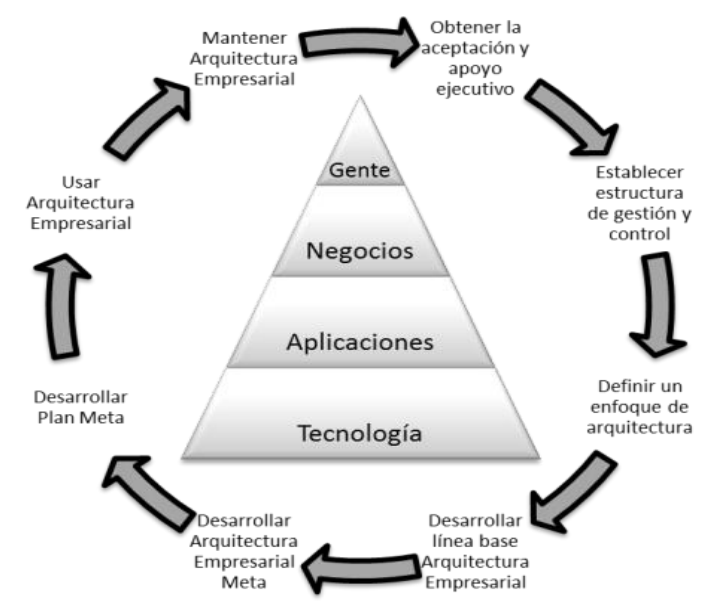

Figura 1. Arquitectura Empresarial. Basado en: (Tucker \& Debrosse, 2003)

\subsection{Herramientas de arquitectura empresarial para la gestión del conocimiento}

La realización de una $A E$ requiere definir algunas actividades: diagnosticar el estado actual de la organización, la hoja de ruta para lograr la misión, la visión y la definición del estado futuro de la organización. Todas estas actividades aportan a la generación de la inteligencia empresarial y son fuentes de nuevo conocimiento.

$\mathrm{AE}$ es esencial para organizaciones que requieran adquirir nuevo software para optimizar su valor de negocio y digitalizar el conocimiento. Además, AE apoya el entendimiento y mejora las interrelaciones entre las dimensiones de conocimiento como son: las personas, los procesos de negocio, los sistemas de información y la infraestructura tecnológica (Schekkerman, 2011). 
Hoy en día las organizaciones que no cuentan con un departamento de $A E$ tienen el riesgo de desarrollar o comprar sistemas duplicados, incompatibles, innecesarios o muy costosos de mantener y, lo más crítico, que no estén alineados a la estrategia corporativa. Una meta esencial para que la $A E$ agregue valor a la organización es que su desarrollo, mantenimiento, implementación y visualización sean apoyados con las herramientas de AE correctas.

Las herramientas de $\mathrm{AE}$ son paquetes de software para la visualización y análisis de las relaciones existentes entre los distintos componentes organizacionales (lacob, Jonkers, Quartel, Franken, \& van den Berg, 2013). Para poder elegir la herramienta de AE adecuada se requiere realizar un análisis previo de las necesidades de los interesados, crear una lista de posibles proveedores de estas herramientas, evaluar las herramientas versus las necesidades y especificaciones del cliente y tomar la decisión de compra.

Las herramientas de $A E$ pueden guiar a los ejecutivos de tecnologías de información y comunicación (TIC) y del negocio a dirigir una organización basados en el conocimiento. El conocimiento se genera al crear un mapa de todas las interacciones existentes en los componentes de la organización y al analizar exhaustivamente las capacidades y oportunidades de esas relaciones. Esto sirve para tomar decisiones acertadas en la gestión del portafolio de aplicaciones o de infraestructura tecnológica. Adicionalmente, las herramientas de AE pueden ayudar a impulsar la colaboración entre los profesionales de TIC y del negocio. En otras palabras la $A E$ apoya a los directivos en realizar las actividades empresariales que son necesarias de una forma adecuada y con un riesgo mínimo.

\section{Metodología y resultados}

El objetivo de esta investigación es otorgar claridad a las organizaciones para que puedan elegir una metodología adecuada que podría usarse para seleccionar software de AE. Con una selección correcta se proyecta contar con una herramienta que permita gestionar el conocimiento organizacional y que se integre al software existente. El método usado en este trabajo se basa en la realización de un caso de estudio en una organización logística. Esta organización tiene la necesidad de adquirir una herramienta de $\mathrm{AE}$ que le permita digitalizar su conocimiento. Además, en este artículo se detallan estudios que listan las varias herramientas de $A E$ que existen en el mercado y se presenta un enfoque para la digitalización del conocimiento organizacional.

Uno de los mayores desafíos de AE es el de ser capaz de proveer soporte a las decisiones de acuerdo a las metas y estrategias. En este contexto el análisis de los modelos que entrega la $A E$ otorga la visibilidad necesaria para la toma de decisiones acertadas. Además, analizar los modelos de AE con las herramientas adecuadas puede ser clave para la creación de conocimiento explícito (Johnson, Lagerstrom, Narman, \& Simonsson, 2007). 
Los modelos de $A E$ describen la gente, los procesos de negocio, las aplicaciones y la infraestructura organizacional y las relaciones entre sí. Estos modelos son equivalentes a mapas y constituyen los elementos clave para entender, analizar y diseñar una organización, sus sistemas de información y su infraestructura tecnológica. Por lo tanto, estos modelos son fundamentales para la creación y la GC (Narman, Johnson, \& Nordstrom, 2007).

El conocimiento que generan las organizaciones se encuadra en tres dimensiones: personas, procesos y tecnología. Una de las tareas más importantes de la GC es buscar mecanismos para traducir y capturar el conocimiento tácito que reside en las personas y en los procesos y convertirlo en conocimiento explícito que pueda ser observado o analizado a través de medios digitales, es decir que las habilidades, capacidades (know-how) de las personas pueda ser transferido a futuros empleados lo cual reduciría los costos de rotación de personal. Es ahí donde radica la importancia de la $A E$ ya que esta herramienta permite cumplir con este objetivo. Por otro lado, se encuentra la información que es capturada de los sistemas operacionales del negocio. Esta información requiere de un análisis exhaustivo aplicando diferentes técnicas de minería de datos o inteligencia de negocios para obtener conocimiento.

\section{Caso de Estudio}

Está investigación fue realizada en cooperación con un proveedor de servicios logísticos (PSL) con presencia internacional. El objetivo principal del estudio fue el de evaluar y seleccionar una herramienta de $\mathrm{AE}$ que permita digitalizar el conocimiento en este PSL. Las actividades realizadas, validadas y que se sugieren realizar para la digitalización del conocimiento en una organización son las siguientes:

1. Análisis de departamentos e interesados.

2. Evaluación y selección de la herramienta de AE.

3. Digitalización del conocimiento.

4. Mejora de los procesos.

\subsection{Análisis de departamentos e interesados}

El PSL donde se realizó el caso de estudio es una empresa con presencia a nivel mundial. La identificación de los interesados puede llevarse a cabo usando la matriz de poder-interés (Ackermann \& Eden, 2011). Esta matriz apoya la decisión de los interesados que deben ser entrevistados de acuerdo al interés y poder que tengan en el proyecto. La matriz se muestra en la Figura 2.

Esta matriz tiene 4 cuadrantes donde se deben ubicar los interesados. Por ejemplo, para la digitalización del conocimiento del departamento de software, el programador tendría un alto 
interés pero un poder mínimo en las decisiones que se tienen que tomar. Por lo cual, se lo debería ubicar en el cuadrante alto interés y bajo poder (Mantener Informadas). Al ubicarlo en este cuadrante podemos gestionar mejor las comunicaciones con el interesado y podremos saber si es un recurso clave para las entrevistas de levantamiento y digitalización de procesos. Los principales interesados identificados en el proyecto son los arquitectos de negocio y tecnología.

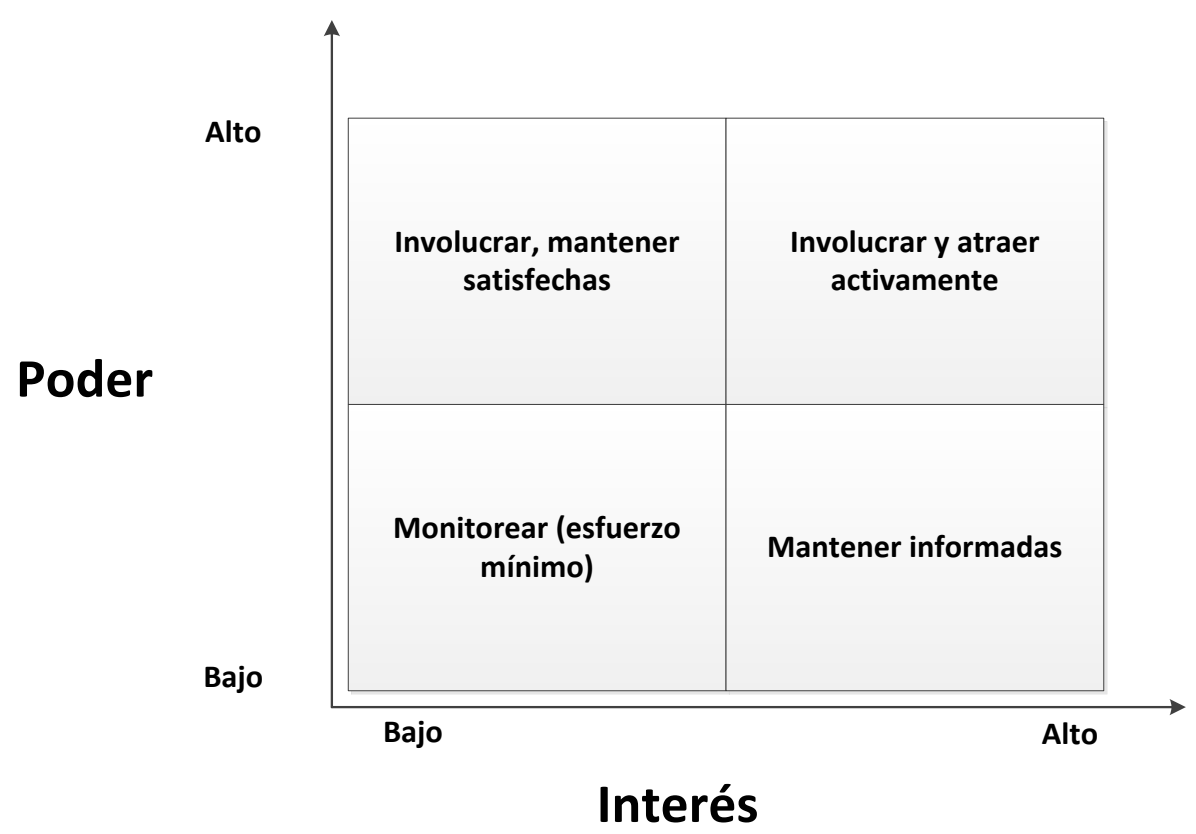

Figura 2. Matriz de interesados poder-interés

Algunos de los requerimientos de los interesados para el software de AE fueron:

1. La herramienta de software de AE debe ser considerada como líder en el mercado.

2. La herramienta debe proveer fuertes capacidades para la integración con los sistemas de información existentes.

3. La herramienta debe proveer fuertes capacidades de análisis y reportes

\subsection{Evaluación y selección de herramienta de arquitectura empresarial}

Es importante empezar la evaluación realizando una investigación bibliográfica para identificar las herramientas de $\mathrm{AE}$ existentes en el mercado. Las herramientas de $\mathrm{AE}$ permiten digitalizar el conocimiento al poder transformar el conocimiento implícito en modelos que describen las estructuras organizacionales (personas), los procesos del negocio, las aplicaciones y la tecnología. La mayoría de las herramientas de $\mathrm{AE}$ se basan en el estándar Archimate (Schekkerman, 2011). El lenguaje Archimate permite diseñar arquitecturas en diferentes dominios y la creación de relaciones entre los distintos objetos de la organización. 
En el caso de estudio realizado se identificaron las siguientes fuentes que listan herramientas de AE: encuesta de herramientas de gestión de AE de la Universidad Técnica de Münich (F. Matthes, Buckl, Leitel, \& Schweda, 2008), la guía de selección de herramientas de AE del Instituto de Desarrollo de AE (Schekkerman, 2011) y la encuesta de herramientas de AE de la empresa Syracom (Knoll \& Schulz, 2013). En total se identificó 46 posibles herramientas de AE. Adicionalmente, existen algunas iniciativas de evaluación de herramientas de $A E$ que nos permiten tener indicadores de cuál podría ser la herramienta adecuada para gestionar el conocimiento en nuestra organización.

Dos fuentes reconocidas de evaluación son: el cuadrante mágico de Gartner (en inglés Gartner Magic Quadrant) y la curva de Forrester (en inglés Forrester Wave). En el cuadrante mágico de Gartner del año 2016 se destaca como líderes a: Mega, Alphabet y Bizzdesign Enterprise Studio (Gartner Inc., 2016). En cuanto a la curva de Forrester, la última publicada es del 2015, la misma ubica a Alphabet y a Orbus software como líderes (Forrester Research, 2015).

Posterior a esto se debe realizar un preprocesamiento, limpieza y filtrado de información para descartar aquellas herramientas que hayan salido del mercado y aquellas cuyas empresas comercializadoras hayan sido adquiridas o fusionadas. Como resultado de esta fase se debe obtener una lista larga de herramientas que serán evaluadas. La lista larga depende de la cantidad total de herramientas existentes en el mercado, para el campo de AE esta puede ser de unas 10 herramientas que se ajusten a los requisitos generales. Para realizar esta evaluación se recomienda usar una metodología de evaluación de software.

Después de investigar las metodologías de evaluación de software que fueron presentados dentro una revisión muy completa por (Jadhav \& Sonar, 2009), se pudo detectar que no existe una metodología específica para la evaluación de herramientas de AE. Es por esto que se analizaron y clasificaron aquellas metodologías que podrían ser utilizadas para la evaluación de herramientas de AE. Está clasificación se basó en los 52 artículos presentados por Jadhav \& Sonar en su artículo.

El trabajo realizado por (Jadhav \& Sonar, 2009) fue el de reducir de 130 a 52 artículos con metodologías de evaluación de software. Esta reducción se realizó en tres pasos. En el primer paso se analizaron los resúmenes de los artículos para descartar aquellos que no fueran relevantes y que no presentasen metodologías completas de evaluación. En el segundo paso se aplicó un filtro en el proceso de extracción de datos para eliminar aquellos artículos que no cumplían con los requerimientos de evaluación de software. En el paso final se descartaron aquellos artículos que describían sistemas o herramientas automáticas de evaluación ya que se salían del alcance de la investigación. 
En un trabajo posterior realizado como parte de esta investigación se redujo el número de artículos de 52 a 14. El criterio de reducción fue el siguiente: descartar las metodologías que se concentran en la evaluación y selección de productos específicos de software ya que la meta de este artículo es sugerir una metodología general que pueda ser adaptada al campo de AE. La especificidad fue primordialmente encontrada en dos categorías: 1. tipo de software (herramientas de ingeniería de software asistido por computador, software de simulación, paquetes de ERP, etc.) y 2. tamaño de la compañía. Las 14 metodologías seleccionadas después de este proceso se muestran en la Tabla 1.

Tabla 1. Metodologías de evaluación de software no específicas

\begin{tabular}{|c|c|c|}
\hline Autor(es) & Año & Tipo de Software \\
\hline Bhuta y Boehm & 2005 & Componentes de Software Comercial \\
\hline Comella-Dorda et al & 2004 & Software Comercial \\
\hline Franch y Carvallo & 2003 & Paquetes de Software \\
\hline Kontio et al & 1995 & Software Comercial \\
\hline Kunda & 2003 & Componentes de Software Comercial \\
\hline Lawlis et al. & 1997 & Software Comercial \\
\hline Lee et al & 2003 & Selección de Software \\
\hline Leung et al. & 2002 & Software Comercial \\
\hline Morera & 2002 & Software Comercial \\
\hline Morisio y Tsoukias & 1997 & Software Comercial \\
\hline Oh and Lee & 2003 & Componentes de Software Comercial \\
\hline Sanders et al. & 1983 & Paquetes de Software de Computación \\
\hline Toshtzar & 1988 & Software de Computación \\
\hline Welzel y Hausen & 1993 & Producto de Software \\
\hline
\end{tabular}

En una etapa consecuente se analizó detalladamente los resúmenes de los 14 artículos resultantes. Durante esta fase otro grupo de artículos fue descartado. Los criterios usados para descartar los artículos fueron los siguientes: artículos que no incluían una metodología de selección, artículos que requerían análisis de otros componentes organizacionales y artículos que requerían de un ambiente complejo de evaluación por ejemplo: muchos evaluadores, evaluación de todas las herramientas del mercado, evaluación de la arquitectura del sistema, creación de modelos de evaluación basados en la organización, artículos basados en microprocesamiento y microprogramación. Luego de excluir los artículos con los filtros previamente mencionados, la lista final de artículos con metodologías que son consideradas para la evaluación y selección de herramientas de $\mathrm{AE}$ se redujo a tres como se muestra en la Tabla 2. 
Tabla 2. Metodologías de evaluación de software para AE

\begin{tabular}{|c|c|c|}
\hline Autor(es) & Año & Tipo de Software \\
\hline (Comella-Dorda et al., 2004) & 2004 & Software Comercial \\
\hline (Kontio, Chen, \& Limperos, 1995) & 1995 & Software Comercial \\
\hline (Morera, 2002) & 2002 & Software Comercial \\
\hline
\end{tabular}

Para evaluar y seleccionar una herramienta de $A E$ es importante formar un equipo de evaluación que conozca del negocio y los objetivos que se busca con AE. Además, es muy valioso que el equipo de evaluación conozca de la infraestructura tecnológica ya que se puede integrar bases de datos de gestión de configuración de infraestructura en inglés Configuration Management Database (CMDB) y software de gestión de procesos con la nueva herramienta de $A E$ lo que disminuirá el tiempo de modelamiento e implementación de las arquitecturas a futuro. Se sugiere elegir una de las metodologías de la Tabla 2 de acuerdo a las necesidades de evaluación y a la comparación realizada en la Tabla 3 . Como se puede ver en la tabla 3 se compara si la metodología presenta una técnica para definir criterios, Comella Dorda et al. y Kontio et al. proponen el uso de la técnica conocida en el inglés como Goal Question Metric (GQM), está técnica permite orientar de mejor manera las metas o criterios que se buscan en una evaluación. Otros parámetros que se comparan son las técnicas de evaluación, metodologías de selección, esfuerzo de implementación y si describen o presentan plantillas para realizar el trabajo de evaluación. Del estudio realizado se puede determinar que (Comella-Dorda et al., 2004) es la que presenta más técnicas de evaluación y un método robusto de evaluación.

Tabla 3. Comparación de metodologías seleccionadas de evaluación de software

\begin{tabular}{|c|c|c|c|c|c|c|}
\hline Autor & Año & Técnica de Criterio & $\begin{array}{l}\text { Técnica de } \\
\text { Evaluación }\end{array}$ & $\begin{array}{l}\text { Metodología de } \\
\text { Selección }\end{array}$ & Plantillas & Esfuerzo \\
\hline $\begin{array}{c}\text { Comella- } \\
\text { Dorda et } \\
\quad \text { al }\end{array}$ & 2004 & Goal Question Metric & $\begin{array}{c}\text { Análisis de } \\
\text { Sensibilidad, } \\
\text { Análisis de Brecha, } \\
\text { Costo de } \\
\text { Cumplimiento, } \\
\text { Promedios de Peso }\end{array}$ & PECA & SI & MEDIO \\
\hline $\begin{array}{c}\text { Kontio et } \\
\text { al }\end{array}$ & 1995 & Goal Question Metric & $\begin{array}{c}\text { Promedios de } \\
\text { Pesos, Proceso } \\
\text { Analítico } \\
\text { Jerárquico } \\
\end{array}$ & OTSO & SI & MEDIO-ALTC \\
\hline Morera & 2002 & NO & $\begin{array}{c}\text { Proceso Analítico } \\
\text { Jerárquico }\end{array}$ & DESMET & NO & MEDIO-ALTC \\
\hline
\end{tabular}

\subsection{Digitalización del conocimiento}

En el caso de estudio la primera actividad realizada fue elegir una metodología de evaluación que se ajuste a los objetivos de la organización y que permita cumplir con los requerimientos de los interesados (stakeholders). La metodología seleccionada en el PSL fue (Comella-Dorda et al., 2004). La decisión se basó en los siguientes factores: el método de selección PECA provee 
diferentes técnicas que pueden ser adaptadas a diferentes escenarios, además, proporciona plantillas para realizar la evaluación en todas sus fases y el esfuerzo requerido en la evaluación es medio. Finalmente, el autor define que el proceso puede ser adaptable: "Espere adaptar este proceso a su propia situación" (Comella-Dorda et al., 2004).

Esta metodología permitió evaluar las herramientas de AE candidatas para la organización y elegir la que presente mejores resultados en la evaluación. Una de las actividades importantes en la evaluación fue el de revisar cómo integrarse a herramientas de CMBD y de gestión de procesos que la organización posee para no tener que modelar todos los objetos y relaciones existentes en su infraestructura.

Luego de tener la herramienta de AE para la digitalización del conocimiento se sugiere definir un proceso para capturar el conocimiento de los empleados de la organización. No existe un enfoque estándar, pero se propone crear talleres, reuniones o entrevistas con el personal responsable de los procesos. Ayuda mucho modelar mientras se llevan a cabo las reuniones o talleres por lo cual es importante contar con un equipo de al menos 2 personas en las entrevistas: el entrevistador y el modelador. El modelamiento implica capturar las actividades que realiza el personal, las aplicaciones que son usadas, y la infraestructura que las soporta.

\subsection{Mejora de los procesos}

Después de digitalizar el estado actual de los procesos es importante aprovechar las reuniones de levantamiento de información y realizar lluvia de ideas por áreas para conceptualizar y sugerir mejoras, estandarizar y eliminar redundancias. En esta etapa se crea modelos del estado futuro de las arquitecturas. Este estado futuro es la meta que tiene la organización en la búsqueda hacia la mejora continua. Se debe definir también un camino a seguir (hoja de ruta) para alcanzar los objetivos propuestos. Es importante también definir áreas prioritarias para que la $A E$ pueda otorgar valor en etapas tempranas del proyecto.

\section{Conclusiones}

La AE juega un rol sumamente importante como herramienta de GC en organizaciones. El principal aporte de este artículo es el de presentar 3 metodologías de evaluación de software que pueden ser usadas en la selección de software de AE.

Las herramientas de AE son indispensables para poder crear arquitecturas de los diferentes dominios empresariales. Estas herramientas apoyan a los ejecutivos en la toma de decisiones ya que analizan la información de todos los componentes organizacionales y sus interrelaciones.

Luego de elegir la herramienta adecuada el siguiente paso es implementarla dentro de la organización. En este trabajo también se presenta un enfoque para digitalización del conocimiento 
con el uso de estas herramientas. Esta tarea ayuda a transformar el conocimiento tácito de los empleados en conocimiento explícito que permita el análisis de esta información.

El software elegido debe alinearse completamente a los requisitos de los directivos de la empresa y a las metas del proyecto de GC, por lo cual la evaluación debe realizarse con una metodología que se adapte al campo de AE como las que se sugiere en este artículo.

\section{Bibliografía}

Ackermann, F., \& Eden, C. (2011). Strategic Management of Stakeholders: Theory and Practice. Long Range Planning, 44(3), 179-196. http://doi.org/10.1016/j.Irp.2010.08.001

Alavi, M., \& Leidner, D. E. (2001). Knowledge Management and Knowledge Management Systems. MIS Quarterly, 25(1), 107-136.

Bricknal, R., Darrell, G., Nilsson, H., \& Pessi, K. (2011). Enterprise Architecture: Critical Factors affecting modelling and management. Retrieved from http://pdf.aminer.org/000/245/735/enterprise_architecting_critical_problems.pdf

Campbell, H. M. (2006). The role of organizational knowledge management strategies in the quest for business intelligence. 2006 IEEE International Engineering Management Conference, 231-236.

Comella-Dorda, S., John, D., Lewis, G., Edwin, M., Patricia, O., \& Erin, H. (2004). A Process for COTS Software. CMU/SEI-2003-017, (July), 17.

Curado, C. (2006). The knowledge-based view of the firm. Instituto Superior de Economia E Gestao, (1959), 18.

de Vries, M., \& van Rensburg, A. (2008). Enterprise Architecture - New business value perspectives. Southafrican Journal of Industrial Engeneering, 19, 1-16.

Edwards, J. (2011). A Process View of Knowledge Management : It Ain't what you do , it's the way that you do it. Journal of Knowledge Management, 9(4), 297-306.

Forrester Research. (2015). Forrester Wave: Enterprise Architecture Management Suites. Retrieved November 30, 2015, from https://www.forrester.com/report/The+Forrester+Wave+Enterprise+Architecture+Management +Suites+Q3+2015/-/E-RES115345

Gartner Inc. (2013). Gartner IT Glossary. Retrieved March 18, 2013, from http://www.gartner.com/it-glossary/enterprise-architecture-ea/

Gartner Inc. (2016). Gartner 2016 Magic Quadrant for Enterprise Architecture Tools. Retrieved August 20, 2016, from http://www.mega.com/en/resource/gartner-2016-magic-quadrantenterprise-architecture-tools

lacob, M.-E., Jonkers, H., Quartel, D., Franken, H., \& van den Berg, H. (2013). Delivering 
Enterprise Architecture with TOGAF and Archimate. (BIZZdesign BV, Ed.). Enschede NL.

Ivanov, K. (2009). 10 Definitions of Enterprise Architecture. Retrieved October 6, 2012, from http://www.ariscommunity.com/users/koiv/2009-08-20-10-definitions-enterprise-architecturewhich-corresponds-yours

Jadhav, A. S., \& Sonar, R. M. (2009). Evaluating and selecting software packages: A review. Information and Software Technology, 51(3), 555-563. http://doi.org/10.1016/j.infsof.2008.09.003

Johnson, P., Lagerstrom, R., Narman, P., \& Simonsson, M. (2007). Enterprise architecture analysis with extended influence diagrams. Information Systems Frontiers, 9(2), 163-180.

Karemente, K., Aduwo, J. R., Mugejjera, E., \& Lubega, J. (2009). Knowledge Management Frameworks. Strengthening the Role of ICT in Development, 35-57.

Knoll, R., \& Schulz, C. (2013). Enterprise Architecture Tool Survey 2013. Wiesbaden. Retrieved from http://www.syracom.de/

Kontio, J., Chen, S., \& Limperos, K. (1995). A cots selection method and experiences of its use. In Proceedings of the 20th Software Engineering Workshop (pp. 486-489).

Lankhorst, M. (2009). Enterprise Architecture at Work Modelling Communication and Analysis (2nd ed.). Berlin Heidelberg: Springer-Verlag.

Matthes, D. (2011). Enterprise Architecture Kompendium. Springer-Verlag.

Matthes, F., Buckl, S., Leitel, J., \& Schweda, C. M. (2008). Enterprise Architecture Management Tool Survey 2008.

Morera, D. (2002). COTS Evaluation Using Desmet Methodology $\{\&\}$ Analytic Hierarchy Process (AHP). In M. Oivo \& S. Komi-Sirviö (Eds.), 4th International Conference, PROFES 2002 Rovaniemi, Finland, December 9--11 (pp. 485-493). Berlin, Heidelberg: Springer Berlin Heidelberg.

Moscoso-Zea, O., Luján-Mora, S., Schweimanns, N., \& Esquetini, C. (2016). Knowledge Management Framework using Enterprise Architecture and Business Intelligence. 18th International Conference on Enterprise Information Systems (ICEIS), 244-249.

Narman, P., Johnson, P., \& Nordstrom, L. (2007). Enterprise architecture: A framework supporting system quality analysis. In 11th IEEE Enterprise Distributed Object Computing Conference $130-141$

Proper, H., \& Lankhorst, M. (2014). Towards essential sensemaking. Enterprise Modelling and Information Systems Architectures, 9(1), 5-21.

Rowley, J. (2000). From learning organisation to knowledge entrepreneur. Journal of Knowledge Management, 4(1), 7-15. 
Schekkerman, J. (2011). Enterprise Architecture Tool Selection Guide. Institute for Enterprise Architecture Developments.

The Open Group. (2011). TOGAF ® Version 9.1. Retrieved from http://goo.gl/djuv15

Tucker, R., \& Debrosse, D. (2003). Enterprise Architecture Roadmap for Modernization. Enterprise Modernization Issue, 7(2).

Wernerfelt, B. (1984). A Resource-based View of the Firm. Strategic Management Journal, 5, 171180. 